\title{
Sensory Analysis of Virgin Olive Oils
}

\author{
Innocenzo Muzzalupo, Massimiliano Pellegrino and Enzo Perri
}

Additional information is available at the end of the chapter

http://dx.doi.org/10.5772/51721

\section{Introduction}

The evaluation of the sensory properties and determination of the importance of these properties in consumer product acceptance represent a major accomplishment in sensory analysis.

Most of the sensory evaluation carried out by a trained panel involves measurement in two main areas, difference testing and descriptive analysis. Sensory difference tests are procedures used to determine whether judges can distinguish between two similar stimuli. In terms of food, the two stimuli are two very similar food samples. These evaluations are used to determine whether slight changes occur due to either product reformulation or the change in technological processing. Difference tests are particularly well adapted to the assessment of vegetable oils during their processing, being used to control refining efficiency.

Furthermore, they may be used to measure slight flavour variation determined by changes in storage or packaging. There are many different methods for sensory difference testing. They are described in detail in several texts (Amerine et al., 1965; Lawless and Heymann, 1998; Meilgaard et al., 1999).

Descriptive analysis, the other main area of sensory evaluation, precisely describes and measures the sensory attributes of food. It follows that this can be done only with trained judges. There are various methodologies that can be used. One procedure is the Quantitative Descriptive Analysis (QDA) developed by the Tragon Corporation of Palo Alto, California (Stone et al., 1974). Judges examine the food and list all the relevant sensory properties. They then use standards to obtain agreement on the descriptive terms being used. By using a scaling procedure, the judge estimates the intensity of each sensory attribute considered. The raw data obtained are then evaluated through common statistic techniques.

There are several applications for descriptive analysis: product development and assessment, comparison of the sensory properties of products which cannot be compared 
simultaneously (e.g., fresh olive virgin oil from consecutive years), shelf-life studies, correlation of instrumental and sensory properties, studies of the effects of technological processing on the sensory characteristics of a product, quality assurance, certification of a preset quality standard, etc.

Another branch of sensory analysis is represented by consumer science. Generally, people eat and drink food because they like it. But, food intake is not completely driven by hedonic motives. Other factors such as market segmentation, advertising, price, packaging, opinions and beliefs play a role too. The sensory perception of sensory quality is an important factor in motivating consumer choice. It derives from the intrinsic properties of the product, perceived by the consumer at the moment of buying (colour, shape, aspect, etc) and subsequently from direct individual experiences (odour, taste). The measurement of liking is necessary before a product is launched onto the market, with substantial capital being needed. This can therefore save investing in a product that may not be liked due to a deficiency of sensory qualities.

The most frequently used method to measure acceptability and preference is the 9-point hedonic scale, developed in 1955 (Jones et al., 1955). The subjects that take part in the sensory testing are not trained, and should be relatively naive to this kind of task.

\section{Sensory analysis of olive oil}

The European Commission (EC) upon issuing the following regulations:

- Commission Regulation n. 2568/91 of 11 July 1991 (EC Regulation, 1991);

- Commission Regulation n. 2472/1997 of 11 December 1997 (EC Regulation, 1997);

- Commission Regulation n. 796/2002 of 06 May 2002 (EC Regulation, 2002);

- Commission Regulation n. 1989/2003 of 06 November 2003 (EC Regulation, 2003);

- Commission Regulation n. 1640/2008 of 04 July 2008 (EC Regulation, 2008);

- Commission Regulation n. 61/11 of 24 January 2011 (EC Regulation, 2011);

set the objective of establishing and developing the criteria needed to evaluate the chemical and sensory characteristics of oil and virgin olive oil as well as the opportune methodology. The introduction of the Panel Test has led to an evolution in the concept of oil quality. The necessary cognitions are set in order to carry out the sensory analysis of virgin olive oil. It tries to standardise the behaviour and procedures of the tasters, who should take into consideration not only the more general indications but also those specific for tasting olive oil. These procedures have led to an evaluation sheet being drawn up by the International Olive Oil Council (IOOC), leading to results being obtained from different Panels, in different areas of the same country as well as different countries to be compared.

Since 1991, this methodology has been part of the regulations of the European Commission for classifying oils and is described in detail in Appendix II of the EU Regulation n. 2568/91.

The method, Quantitative Descriptive Analysis (QDA), defines the main attributes of oil, both positive as well as negative. Appearance as colour was not selected as a quality parameter of virgin olive oil, as a specific dark-coloured glass is used. The official evaluation 
sheet used within the European Union to establish the sensory profile of virgin olive oil is shown below (Fig. 1). The values, expressed as centimetres, are statistically processed to calculate the median of each positive and negative characteristic.

COI/T.20/Doc. No 15/Rev. 3

page 11

Figure 1

PROFILE SHEET FOR VIRGIN OLIVE OIL

INTENSITY OF PERCEPTION OF DEFECTS:
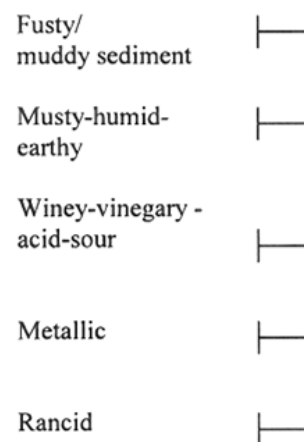
Sensory analysis is an essential technique to characterize food and investigate consumer preferences. International cooperative studies, supported by the International Olive Oil Council (IOOC) have provided a sensory codified methodology for virgin olive oils, known as the "COI Panel test". Such an approach is based on the judgments of a panel of technicians, conducted by a panel leader, who has sufficient knowledge and skills to prepare sessions of sensory analysis, motivate judgement, process data, interpret results and draft the report.

The panel generally consists of a group of 8 to 12 persons, selected and trained to identify and measure the intensity of the different positive and negative sensations perceived.

Sensory assessment is carried out according to codified rules, in a specific tasting room, using controlled conditions to minimize external influences, using a proper tasting glass and adopting both a specific vocabulary and a profile sheet that includes positive and negative sensory attributes. Collection of the results and statistical elaboration must be standardized. The colour of virgin olive oil, which is not significantly related to its quality, may produce expectations and interferences in the flavour perception phase. In order to eliminate any prejudices that may affect the smelling and tasting phases, panel lists use a dark-coloured (blue or amber-coloured) tasting glass.

The panel leader is the person responsible for selecting, training and monitoring tasters to ascertain their level of aptitude according to (IOOC/T.20/Doc.15/rev.3). The number of candidates is generally greater than that needed in order to select people that have a grater sensitivity and discriminatory capability. Screening criteria of candidates are founded on sensory capacity, but also on some personal characteristics of candidates. Given this, the panel leader will personally interview a large number of candidates to become familiar with their personality and understand habits, hobbies, and interest in the food field. He uses this information to screen candidates and rejects those who show little interest, who are not readily available or who are incapable of expressing themselves clearly.

The determination of the detection threshold of the group of candidates for characteristic attributes is necessary because the "threshold concentration" is a point of reference common to a "normal group" and may be used to form homogeneous panels on the basis of olfactorygustatory sensitivity.

A selection of tasters is made by the intensity rating method, as described by Gutiérrez Rosales (Gutiérrez Rosales et al., 1984). A series of 12 samples is prepared by diluting a virgin olive oil characterized by a very high intensity of a given attribute in an odourless and tasteless medium (refined oil or paraffin). The panel leader sends out the candidate, removes one of the 12 tasting glasses from the series, and places the remaining together; the candidate is called back in the room and is asked to correctly replace the testing glass withdrawn from the series by comparing the intensity of this last with that of the others. The test is carried out for fusty, rancid, winey and bitter attributes to verify the discriminating capacity of the candidate on the entire scale of intensities.

The stage training of technicians is necessary to familiarize tasters with the specific sensory methodology, to heighten individual skill in recognizing, identifying and quantifying the sensory attributes and to improve sensitivity and retention with regards to the various 
attributes considered, so that the end result is precise and consistent. In addition, they learn to use a profile sheet.

The maintenance of the panel is made through continuous training during the entire life of the same panel, the check of the sensory acuity of tasters, and exercises that allow the measurement of panel performance.

Every year, every panel must assess a number of reference samples in order to verify the reliability of the results obtained and to harmonize the perception criteria; they must also update the Member State on their activity and on composition changes of their group.

\section{Test conditions}

The test conditions for tasting are described in COI/T.20/Doc. No. 15/Rev. 3:

"The oil sample for analysis shall be presented in standardised tasting glasses conforming to the standard COI7T.20/Doc. No. 5 (Fig. 2).

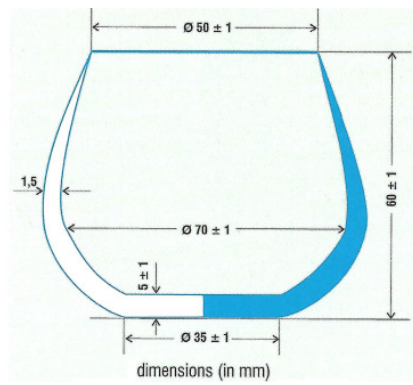

Figure 2. Tasting glass

The glass shall contain 14-16 ml of oil, or between 12.8 and $14.6 \mathrm{~g}$ if the samples are to be weighed, and shall be covered with a watch-glass. Each glass shall be marked with a code made up of digits or a combination of letters and digits chosen at random.

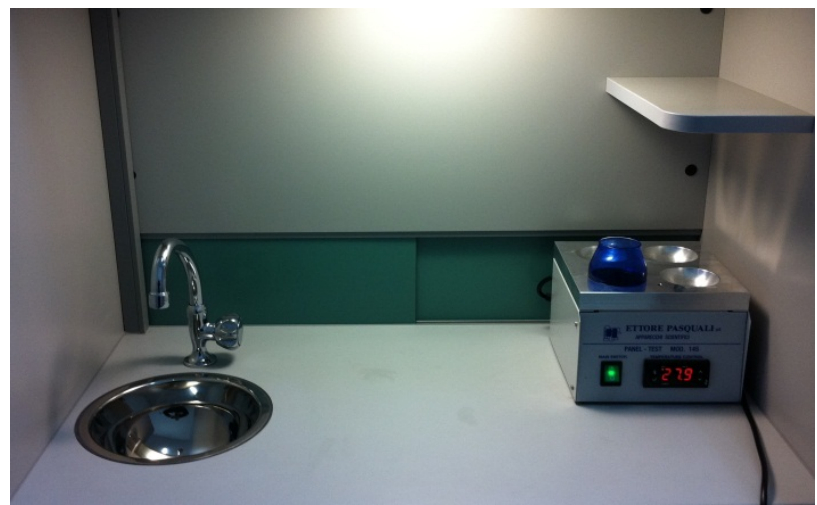

Figure 3. Heating the samples when in the glass 
The oil samples intended for tasting shall be kept in the glass at $28{ }^{\circ} \mathrm{C} \pm 2{ }^{\circ} \mathrm{C}$ throughout the test (Fig. 3).

This temperature has been chosen because it makes it easier to observe organoleptic differences than at ambient temperature and because at lower temperature the aromatic compounds peculiar to these oils volatilize poorly while higher temperatures lead to the formation of volatile compounds peculiar to heated oils. The test room must be at a temperature between 20 and $25^{\circ} \mathrm{C}$.

The morning is the best time for tasting oils. It has been proved that there are optimum perception periods with regards to taste and smell during the day. Meals are preceded by a period in which olfactory-gustatory sensitivity increases, whereas afterwards this perception decreases. However, this criterion should not be taken to the extreme where hunger may distract the tasters, thus decreasing their discriminatory capacity; therefore, it is recommended to hold the tasting session between 10:00 in the morning and 12:00 noon (Fig. 4).

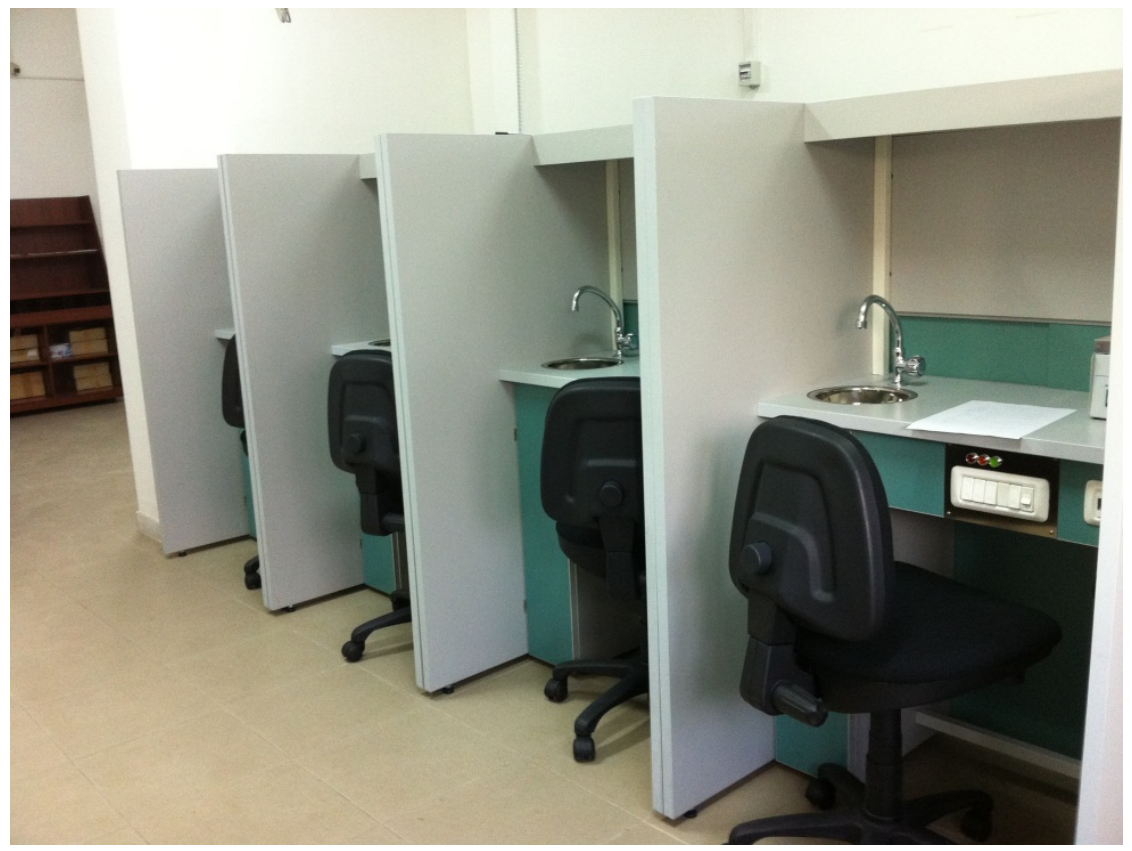

Figure 4. Cabins of the test room

The following recommendations apply to the conduct of the tasters during their work.

When called by the panel leader to participate in an organoleptic test, tasters should be able to attend at the time set beforehand and shall observe the following:

- $\quad$ they shall not smoke or drink coffee at least 30 minutes before the time set for the test;

- they must not have used any fragrance, cosmetic or soap whose smell could linger until the time of the test; 
- $\quad$ they shall fast at least one hour before the tasting is carried out;

- $\quad$ should they feel physically unwell, and in particular if their sense of smell or taste is affected, or if they are under any psychological effect that prevents them from concentrating on their work, the tasters shall refrain from tasting and shall inform the panel leader accordingly;

- when they have complied with the above, the tasters shall take up their place in the booth allotted to them in an orderly, quiet manner;

- $\quad$ they shall carefully read the instructions given on the profile sheet and shall not begin to examine the sample until fully prepared for the task they have to perform (relaxed and unhurried). If any doubts should arise, they should consult the panel leader in private;

- $\quad$ they must remain silent while performing their tasks;

- $\quad$ they must keep their mobile phone switched off at all times to avoid interfering with the concentration and work of their colleagues.

\section{Technique for tasting of virgin olive oil}

The technique applied for tasting is the one described in annex XXI of (EEC) Reg. N. 2568/91:

"the taster shall pick up the glass (Fig. 2), keeping it covered with the watch glass, and shall bend it gently fully in this position so as to wet the inside as much as possible.

Once this stage is completed, he shall remove the watch-glass and smell the sample taking even, slow deep breaths until he has formed a criterion on the oil under assessment. Smelling shall not exceed 30 seconds. If no conclusion has been reached during this time, he shall take a short rest before trying again (Fig. 5).

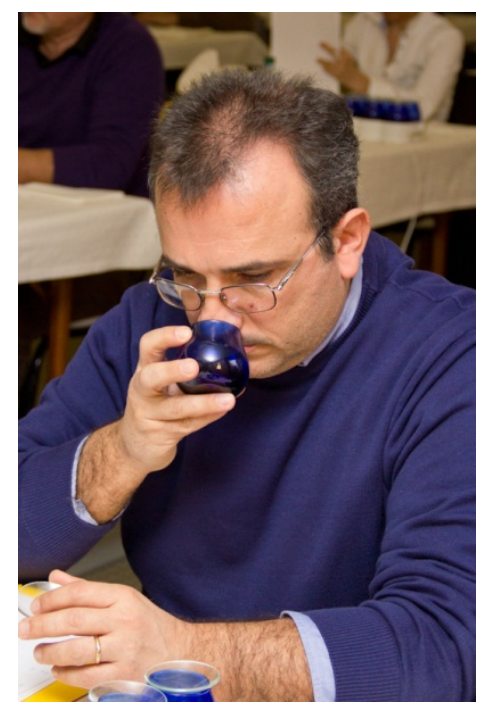

Figure 5. The olfactory test during 
When the olfactory test has been performed, the taster shall then judge the flavour (overall olfactory-gustatory-tactile sensation). To do so, he shall take a small sip of approximately 3 $\mathrm{ml}$ of oil. It is very important to distribute the oil throughout the whole of the mouth cavity, from the front part of the mouth and tongue along the sides to the back part and to the palate support, since it is a known fact that the perception of the four primary tastes, sweet, salty, acid and bitter varies in intensity depending on the area of the tongue and palate.

It should be stressed that it is essential for a sufficient amount of the oil to be spread very slowly over the back of the tongue towards the throat while the taster concentrates on the order in which the bitter and pungent stimuli appear; if this is not done, both of these stimuli may escape notice in some oils or else the bitter stimulus may be obscured by the pungent stimulus.

Taking short, successive breaths, drawing in air through the mouth, enables the taster not only to spread the sample extensively over the whole of the mouth but also to perceive the volatile aromatic components via the back of the nose.

Tactile sensation shall also be taken into consideration. Consequently, fluidity, stickiness and sharpness or sting shall be noted down when detected, and if required for the test, their intensity shall be quantified.

When organoleptically assessing a virgin olive oil, only one sample shall be evaluated in each session to avoid the contrast effect that could be produced by immediately tasting other samples.

As successive tasting produces fatigue or loss of sensitivity, it is important to use a product that can eliminate the remains of the oil from the preceding tasting from the mouth.

The use of a small slice of green apple (about $15 \mathrm{~g}$ ) is recommended which, after being chewed, can be spat out into a spittoon. Then the mouth should be rinsed out with a little water at ambient temperature. At least 15 minutes should lapse between the end of one session and the start of the next.

The panel leader will collect the profile sheets completed by each taster and review the intensities assigned to the different attributes. Should they find any anomaly, they shall invite the taster to revise his or her profile sheet and, if necessary, to repeat the test.

The panel leader will enter the assessment data of each panel member in a computer program like that appended to this method with a view to statistically calculating the results of the analysis, based on their median.

\section{Definition and classification of olive oil.}

Virgin olive oil is the oil obtained from the fruit of the olive tree either by mechanical or other physical means under conditions, particularly thermal conditions, that do not lead to 
alterations in the oil, and which has not undergone any treatment other than washing, decantation, centrifugation and filtration.

In Table 1, the various classes of olive oil as divided by European legislation are reported. The subdivisions in different classes are based on the degree of acidity as well as other analytical parameters and sensory evaluation indices.

1. Extra virgin olive oil: is the virgin olive oil which has a free acidity, expressed as oleic acid, of not more than 0.8 gram per 100 grams, and the sensory characteristics with median defects is 3.5 and the median of fruitiness greater than 0 .

2. Virgin olive oil: is the virgin olive oil which has a free acidity, expressed as oleic acid, of not more than 2 grams per 100 grams and the sensory characteristics with median defects greater than 0 , but less than or equal to 2.5 and the median of fruitiness greater than 0 .

3. Lampante olive oil: is the virgin olive oil which has a free acidity, expressed as oleic acid, of more than 2 grams per 100 grams and the sensory characteristics with median defects greater than 3.5 or if the median defects is less than or equal to 3.5 and the median of fruitiness is 0 . Such olive oil is intended for refining purposes.

4. Refined olive oil: is the olive oil obtained from lampante olive oils by refining methods which do not lead to alterations in the initial glyceridic structure, which has a free acidity, expressed as oleic acid, of less than or equal to 0.3 grams per 100 grams.

5. Blended olive oil: is the oil consisting of a blend of refined olive oil and virgin olive oil which has a free acidity, expressed as oleic acid, of less than or equal to 1 grams per 100 grams. It can be used for human consumption.

6. Crude olive-pomace oil: is the oil obtained by treating olive pomace with solvents, to the exclusion of oils obtained by re-esterification processes and of any mixture with oils of other kinds. This oil is intended for refining with a view to its use in food for human consumption.

7. Refined olive-pomace oil: is the oil obtained from crude olive-pomace oil by refining methods which do not lead to alterations in the initial glyceridic structure, which has a free acidity, expressed as oleic acid, of less than or equal to 0.3 grams per 100 grams.

8. Olive-pomace oil: is the oil comprising the blend of refined olive-pomace oil and virgin olive oil which has a free acidity, expressed as oleic acid, of less than or equal to 1 gram per 100 grams. It can be used for consumption, but in no case should this blend be called "olive oil".

One important consideration is that the first two oils are the best for human consumption due to them being within the set parameters, when obtained directly from the olive press. The third type of oil cannot be consumed until it has been rectified, which gives the fourth type of oil. A small amount of extra-virgin olive oil or virgin olive oil is added to this rectified oil and is known as "Olive oil". 


\begin{tabular}{|c|c|c|c|c|c|c|c|c|c|c|c|c|}
\hline \multicolumn{13}{|c|}{ OLIVE OIL CHARACTERISTICS } \\
\hline casagery & 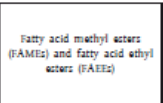 & $\begin{array}{c}\text { Acdity } \\
\text { की) } \\
\text { के }\end{array}$ & 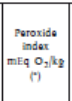 & 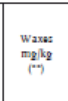 & 2-2yjecerl monopalmiato & 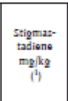 & 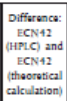 & $\mathrm{k}_{2}, 0$ & $\mathrm{~K}_{20} 017$ & Detatex of & 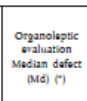 & 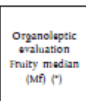 \\
\hline 1. Extra virgin olive oil & 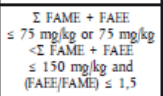 & $\leq 0,8$ & $\leq 20$ & $\leq 250$ & $\begin{array}{l}\leq 0,9 \text { if toral palmitic } \\
\text { acid \% } 14 \% \\
\leq 1,0 \text { if toral palmitic } \\
\text { acid \% }>14 \%\end{array}$ & $\leq 0,10$ & $\leq 0,2$ & $\leq 2,50$ & $\leq 0,22$ & $\leq 0,01$ & Md $=0$ & Mf $>0$ \\
\hline 2. Virgin olive oil & - & $\leq 2,0$ & $\leq 20$ & $\leq 250$ & $\begin{array}{l}\leq 0,9 \text { if toal palmitic } \\
\text { acid \% } 14 \% \\
\leq 1,0 \text { if toal palmitic } \\
\text { acid } \%>14 \%\end{array}$ & $\leq 0,10$ & $\leq 0,2$ & $\leq 2,60$ & $\leq 0,25$ & $\leq 0,01$ & Md $\leq 3,5$ & Mf $>0$ \\
\hline 3. Lampanee olive oil & - & $>2,0$ & - & $\leq 300(?)$ & $\begin{array}{l}\leq 0,9 \text { if toal palmitic } \\
\text { acid \% } 14 \% \\
\leq 1,1 \text { if toal palmitic } \\
\text { acid \%>14\% }\end{array}$ & $\leq 0,50$ & $\leq 0,3$ & - & - & - & $\mathrm{Md}>3,5 \mathrm{e})$ & - \\
\hline 4. Refined olive oil & - & $\leq 0,3$ & $\leq 5$ & $\leq 350$ & $\begin{array}{l}\leq 0,9 \text { if toal palmitic } \\
\text { acid \% } 114 \% \\
\leq 1,1 \text { if toal palmitic } \\
\text { acid } \%>14 \%\end{array}$ & - & $\leq 0,3$ & - & $\leq 1,10$ & $\leq 0,16$ & - & - \\
\hline $\begin{array}{l}\text { 5. Olive oil composed of } \\
\text { refined and virgin olive } \\
\text { oils }\end{array}$ & - & $\leq 1,0$ & $\leq 15$ & $\leq 350$ & $\begin{array}{l}\leq 0,9 \text { if toal palmitic } \\
\text { acid } \% \leq 14 \% \\
\leq 1,0 \text { if toal palmitic } \\
\text { acid } \%>14 \%\end{array}$ & - & $\leq 0,3$ & - & $\leq 0,90$ & $\leq 0,15$ & - & - \\
\hline 6. Crude olive-residue oil & - & - & - & $>350(4)$ & $\leq 1,4$ & - & $\leq 0,6$ & - & - & - & - & - \\
\hline 7. Refined olive-residue oil & - & $\leq 0,3$ & $\leq 5$ & $>350$ & $\leq 1,4$ & - & $\leq 0,5$ & - & $\leq 2,00$ & $\leq 0,20$ & - & - \\
\hline 8. Olive-residue oil & - & $\leq 1,0$ & $\leq 15$ & $>350$ & $\leq 1,2$ & - & $\leq 0,5$ & - & $\leq 1,70$ & $\leq 0,18$ & - & - \\
\hline
\end{tabular}

\begin{tabular}{|c|c|c|c|c|c|c|c|c|c|c|c|c|c|c|c|c|}
\hline \multirow[b]{2}{*}{ category } & \multicolumn{6}{|c|}{ Acid consent (1) } & \multirow[b]{2}{*}{ 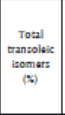 } & \multirow{2}{*}{ 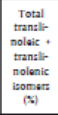 } & \multicolumn{6}{|c|}{ Sterols composition } & \multirow[b]{2}{*}{ 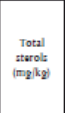 } & \multirow[b]{2}{*}{ 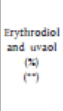 } \\
\hline & 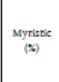 & $\begin{array}{l}\text { Linolenic } \\
\text { (is) }\end{array}$ & $\begin{array}{c}\text { Arachidic } \\
\text { (s) }\end{array}$ & (a) & 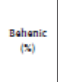 & 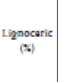 & & & $\begin{array}{l}\text { Cholestersol } \\
(\$) ;\end{array}$ & 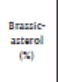 & $\begin{array}{c}\text { campesterol } \\
\text { (is) }\end{array}$ & 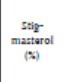 & 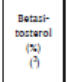 & 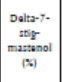 & & \\
\hline 1. Extra virgin olive oil & $\leq 0,05$ & $\leq 1,0$ & $\leq 0,6$ & $\leq 0,4$ & $\leq 0,2$ & $\leq 0,2$ & $\leq 0,05$ & $\leq 0,05$ & $\leq 0,5$ & $\leq 0,1$ & $\leq 4,0$ & $<$ Camp. & $=93,0$ & $\leq 0,5$ & $=1000$ & $\leq 4,5$ \\
\hline 2. Virgin olive oil & $\leq 0,05$ & $\leq 1,0$ & $\leq 0,6$ & $\leq 0,4$ & $\leq 0,2$ & $\leq 0,2$ & $\leq 0,05$ & $\leq 0,05$ & $\leq 0,5$ & $\leq 0,1$ & $\leq 4,0$ & $<$ Camp. & $=93,0$ & $\leq 0,5$ & $=1000$ & $\leq 4,5$ \\
\hline 3. Lampante olive oil & $\leq 0,05$ & $\leq 1,0$ & $\leq 0,6$ & $\leq 0,4$ & $\leq 0,2$ & $\leq 0,2$ & $\leq 0,10$ & $\leq 0,10$ & $\leq 0,5$ & $\leq 0,1$ & $\leq 4,0$ & - & $=93,0$ & $\leq 0,5$ & $=1000$ & $\leq 4,50$ \\
\hline 4. Refined olive oil & $\leq 0,05$ & $\leq 1,0$ & $\leq 0,6$ & $\leq 0,4$ & $\leq 0,2$ & $\leq 0,2$ & $\leq 0,20$ & $\leq 0,30$ & $\leq 0,5$ & $\leq 0,1$ & $\leq 4,0$ & < Camp. & $=93,0$ & $\leq 0,5$ & $=1000$ & $\leq 4,5$ \\
\hline $\begin{array}{l}\text { 5. Olive oil composed of } \\
\text { refined and virgin olive oils }\end{array}$ & $\leq 0,05$ & $\leq 1,0$ & $\leq 0,6$ & $\leq 0,4$ & $\leq 0,2$ & $\leq 0,2$ & $\leq 0,20$ & $\leq 0,30$ & $\leq 0,5$ & $\leq 0,1$ & $\leq 4,0$ & $<$ Camp. & $=93,0$ & $\leq 0,5$ & $=1000$ & $\leq 4,5$ \\
\hline 6. Crude olveresidue oil & $\leq 0,05$ & $\leq 1,0$ & $\leq 0,6$ & $\leq 0,4$ & $\leq 0,3$ & $\leq 0,2$ & $\leq 0,20$ & $\leq 0,10$ & $\leq 0,5$ & $\leq 0,2$ & $\leq 4,0$ & - & $=93,0$ & $\leq 0,5$ & $=2500$ & $>4,519$ \\
\hline 7. Refined olive-residue oil & $\leq 0,05$ & $\leq 1,0$ & $\leq 0,6$ & $\leq 0,4$ & $\leq 0,3$ & $\leq 0,2$ & $\leq 0,40$ & $\leq 0,35$ & $\leq 0,5$ & $\leq 0,2$ & $\leq 4,0$ & < camp. & $=93,0$ & $\leq 0,5$ & $=1800$ & $>4,5$ \\
\hline 8. Olive-residue oil & $\leq 0,05$ & $\leq 1,0$ & $\leq 0,6$ & $\leq 0,4$ & $\leq 0,3$ & $\leq 0,2$ & $\leq 0,40$ & $\leq 0,35$ & $\leq 0,5$ & $\leq 0,2$ & $\leq 4,0$ & $<$ Camp. & 293,0 & $\leq 0,5$ & 21600 & $>4,5$ \\
\hline \multicolumn{17}{|c|}{ 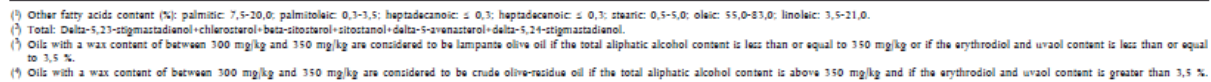 } \\
\hline \multicolumn{17}{|c|}{ 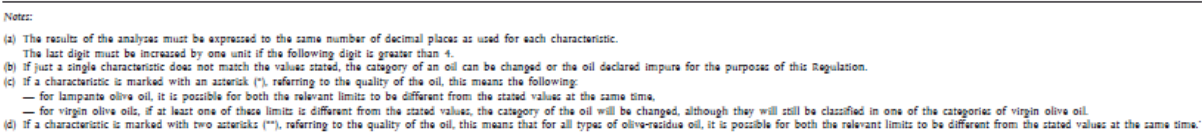 } \\
\hline
\end{tabular}

Table 1. Analytical and sensory properties of olive oils 


\section{Olive oil tasting attributes}

Olive oil as judged by experts shows a multitude of either positive or negative characteristics.

\subsection{Positive attributes}

Almond: light smell recalling that of fresh or dried almond.

Apple: a sensation recalling this fruit.

Artichoke: a smell recalling raw artichoke.

Astringent: a puckering sensation in the mouth created by tannin.

Bitter: this is a preferred characteristic taste of olive oils, if it is not too highly intense.

Fruity: range of smells (dependent on variety) characteristic of oil from healthy fresh fruit, green or ripe, perceived directly and/or retro nasally. Fruitiness is qualified as green if the range of smells is reminiscent of green grass. Fruitiness is qualified as ripe if the range of smells is reminiscent of ripe fruit and is characteristic of oil from green and ripe fruit.

Green grass: a sensation recalling that of freshly cut grass.

Hay: a smell recalling that of dried grass.

Spicy: a tactile sensation similar to that of a light chilli pepper, especially in the back of the throat, which can force a cough.

\subsection{Negative attributes}

Brine: salty taste of oil made from brined olives.

Coarse: a tactile sensation in the mouth due to texture of oil.

Cucumber: off flavour from prolonged storage, particularly in tin.

Dreggiest: odour of warm lubricating oil and is caused by the poor or lack of the decanting process.

Earthy: this term is used when oil has acquired a musty humid odour because it has been pressed from unwashed, muddy olives.

Esparto: hemp-like smell acquired when olive paste has been spread on Esparto mats. Smells may differ according to whether the mates are green or dried.

Hemp: caused by the use of filtering panels, which are not perfectly clean, and recalls hemp. 
Flat: oils which have lost their characteristic aroma and have neither taste nor smell.

Frozen: due to olives which have been exposed to freezing temperatures. When cooked, this oil gives off very unpleasant odours.

Fusty: due to olives fermenting in piles while in storage waiting to be pressed.

Grubby: smell imparted by grubs of the olive fly. The smell is both rotten and putrid at the same time.

Heated: prolonged heating during extraction processing.

Muddy: typical odour of oil that has been stored to long on its own sediment.

Musty: mouldy smell from olives being stored too long before pressing.

Metallic: oils processed or stored with extended contact to metal surfaces.

Rancid: old oils that have started oxidizing due to exposure to light or air.

Vegetable water: oils that have absorbed the unpleasant odours and flavours of the vegetable water after pressing that they have remained in contact for too long.

Wine-vinegar: typical odour of wine or vinegar due to fermentation of olives.

\section{Factors that affect the sensory quality of virgin olive oil}

The evaluation of exactly how various factors affect the sensory quality of the final product is essential in order to distinguish among different types of oil. The following factors all play a role in producing high quality olive oil (Angerosa, 2002; Angerosa et al., 2004, Benincasa et al., 2011).

Cultivar. The numerous varieties constitute an important element for the production of extra virgin olive oils, characterised by different organoleptic characteristics.

Cultivation Techniques (irrigation, fertilization, treatment of plants, diseases etc). Among the environmental factors that influence the quality of the olives and therefore the oil, both the temperature and the amount of water available have an important role, with the first affecting the acidic composition of the olives, while the latter the amount of phenolic substances.

Maturation of the olive. An early harvest generally gives a more bitter and spicy oil due to the high phenol content.

Harvest and Storage of the olive. The quality of the oil is highly conditioned by the state of integrity of the olive. Traditional manual harvesting techniques avoid damaging the fruit in comparison to mechanical methods. Storage of the olives in not very big crates, avoids an excessive mass of olives that could either become crushed or 
overheated, facilitating attacks from micro-organisms as well as oxidation and fermentation.

De-leafing and washing of the olive. Before being processed, the olives must be cleaned of any superfluous material, including leaves, and branches. These are all elements that can negatively influence the quality of the oil.

Pressing. The olives are broken during the pressing phase with the skin and the pulp being lacerated as well as the stone crushed. The press can be a traditional "pan-mill" one, either in a discontinuous system or combined with an extraction system in order to carry out continual centrifugation. These presses can either be hammers or disks. Metal presses have a more violent pressing of the olive (above all hammered ones) as well as a greater laceration of the skin, giving a higher extraction of the phenolic composites and therefore a more bitter and spicy oil that lasts longer.

Kneading. Prolonged kneading and high temperatures could increase the activity of pectoltic and proteolitic enzymes, negatively modifying the chemical-physical characteristics and therefore the quality of the oil.

Extraction. The systems to separate the liquid from the solid can be divided into two groups:

a. mechanical pressure on the paste through a series of operations that make the process discontinuous,

b. by centrifugation that is continuous. The extraction process by pressure has the risk of contaminating the oil due to wear and dirtying of the filters. The continual centrifugation system guarantees greater hygiene and therefore gives oil with elevated qualitative characteristics.

Centrifugation. The oily liquid contains a certain amount of water (called "of vegetation") that is eliminated by centrifuging the product. This operation allows the suspended solid substances to also be eliminated. Water is often added in order to rid the oil of the watery impurities. However, this reduces the phenolic substances content.

Clarification and filtration. The oil obtained from centrifugation still contains mucilage, water and small pieces of the fruit. It is also turbid and opalescent. A clarification process is then carried out in order to eliminate these substances that can favour hydrolysis and/or oxidation. Traditional clarification methods include sedimentation, and have now been substituted by filtration. "Light" filtration systems are preferable rather than more drastic ones that can provoke a reduction in the anti-oxidants and subsequently a reduced shelf-life with the possibility of turning rancid.

Conservation of the oil. In order to maintain both the chemical-physical and organoleptic properties of the oil, the conservation conditions must be controlled. The main factors affecting the conservation of oil are: 
a. the temperature $\left(12-15^{\circ} \mathrm{C}\right)$,

b. light (oil should be stored in the dark otherwise the photo-oxidization process on the polyunsaturated fatty acids can determine the rancid defect),

c. oxygen in the air (a series of oxidation reactions occur when the oil comes into contact with the air, modifying the chemical composition and subsequently the colour, smell and flavour). It is therefore good practice to store extra virgin olive oil in a sealed environment.

\section{Conclusion}

Virgin olive oil is the only product that since 1991 has undergone sensory analysis regulated by European norms. It can be noted that the methodology for the sensory evaluation of virgin olive oil is well documented and standardized. Sensory analysis of olive oil is the evaluation of an oil's organoleptic attributes, which are appreciated through the senses of smell and taste. Sensory analysis is an essential part of evaluating olive oil quality and complements chemical analysis, both of which are requirements for determining the quality of olive oil according to International Olive Council (IOC) and new USDA standards.

The studies on olive oil have highlighted that the sensory qualities are affected by several agronomic and climatic parameters as well as extraction process. Due to here being a variation in the chemical composition of the oil, there is a possibility that these chemical compounds could be considered markers to be used to differentiate the various factors that affect overall quality.

The results of the quantitative descriptive analysis carried out by 9 members of staff of the CRA-OLI on the virgin olive oils produced by single varieties cultivated in the collection field of CRA-OLI are given in the elaiographic cards attached to Chapter "Description of varieties"

\section{Author details}

Innocenzo Muzzalupo*, Massimiliano Pellegrino and Enzo Perri

Agricultural Research Council - Olive Growing and Oil Industry Research Centre, Rende (CS), Italy

\section{Acknowledgement}

The authors thank all tasters and the panel leader of the panel at the CRA-OLI. The author thank the Projects: CERTOLIO, GERMOLI and OLIOPIU' for financial support.

\footnotetext{
${ }^{*}$ Corresponding Author
} 


\section{References}

Amerine, M.A.; Pangborn, R.M. \& Roessler, E.B. (1965). Principles of sensory evaluation of food. Academic Press, New York, ISBN 0120561506.

Angerosa F. (2002). Influence of volatile compounds on virgin olive oil quality evaluated by analytical approaches and sensor panels. European Journal of Lipid Science and Technology, Vol. 104, pp. 639-660, ISSN 1438-9312.

Angerosa, F.; Servili, M; Selvaggini, R.; Taticchi, A.; Esposto, S. \& Montedoro, G.F. (2004). Volatile compounds in virgin olive oil: occurance and their relationship with the quality. Journal of Chromatography A, Vol. 1054, pp. 17-31, ISSN 0021-9673.

Benincasa, C.; Russo, A.; Romano, E.; Elsorady, M.E.; Perri, E. \& Muzzalupo I. (2011). Chemical and sensory analysis of some egyptian virgin olive oils. Journal Nutrition $\mathcal{E}$ Food Sciences, Vol. 1, pp. 1-8, ISSN 0034-6659.

European Community, Commission Regulation (1991) 2568/91. On the characteristics of olive oil and olive residue oil and on the relevant methods of analysis. Official Journal of the European Communities, July 11, L248, 1-83.

European Community, Commission Regulation (1997) 2472/97. Amending Regulation No 2568/91/EEC. Official Journal of the European Communities, December 11, L341, 2539.

European Community, Commission Regulation (2002) 796/2002. Amending Regulation No 2568/91/EEC. Official Journal of the European Communities, May 6, L128, 8-28.

European Community, Commission Regulation (2003) 1989/2003. Amending Regulation No 2568/91/EEC. Official Journal of the European Communities, November 6, L295, 5777.

European Community, Commission Regulation (2008) 640/2008. Amending Regulation No 2568/91/EEC. Official Journal of the European Communities, July 4, L178, 11-16.

European Community, Commission Regulation (2011) 61/2011. Amending Regulation No 2568/91/EEC. Official Journal of the European Communities, January 24, L23, 1-14.

Gutiérrez-Rosales, F.; Risco, M.A. \& Gutiérrez González-Quijano, R. (1984). Selección de catadores mediante el método de clasificación por intensidad. Grasas y Aceites, Vol.35, pp. 310-314, ISSN 0017-3495.

International Olive Oil Council (2007). Glass for oil tasting. IOOC/T.20/Doc. 5/rev.1

International Olive Oil Council (2010). Sensory analysis of olive oil. Method for the organoleptic assessment of virgin olive oil. IOOC/T.20/Doc. No 15/Rev. 3.

Jones, L.V.; Peryam, D.R. \& Thurstone, L.L. (1955). Development of a scale for measuring soldiers' food preferences. Food Research, Vol. 20, pp. 512-520.

Lawless, H.T. \& Heymann, H. (1998). Sensory evaluation of food: principle and practices. Ed. Chapman \& Hall. New York, ISBN 0-412-99441-0.

Meilgaard, M.; Civille, G.V. \& Carr, B.T. (1999). Sensory evaluation techniques. 3rd ed., CRC Press, Boca Raton, Florida, ISBN 0-8493-0276-5. 
Olive Germplasm - The Olive Cultivation, Table Olive and Olive Oil Industry in Italy

Stone, H. \& Sidel, J.L. (2004). Sensory evaluation practise. $3^{\text {nd }}$ ed., Academic Press, San Diego, CA, ISBN 0-12-672690-6. 